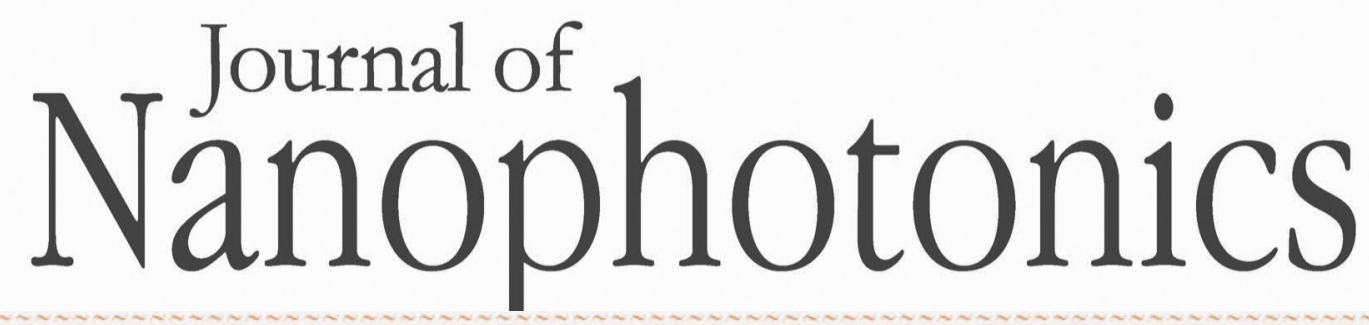

SPIEDigitalLibrary.org/jnp

\title{
DNA as scaffolding for nanophotonic structures
}

Katarzyna Matczyszyn

Joanna Olesiak-Banska 


\title{
DNA as scaffolding for nanophotonic structures
}

\author{
Katarzyna Matczyszyn and Joanna Olesiak-Banska \\ Wroclaw University of Technology, Institute of Physical and Theoretical Chemistry, \\ Wybrzeże Wyspianskiego 27, 50-370 Wrocław, Poland \\ katarzyna.matczyszyn@pwr.wroc.pl
}

\begin{abstract}
Deoxyribonucleic acid (DNA) chains can be engineered for diverse nanophotonics applications by the insertion of molecular groups in different spatial configurations. DNA chains can be assembled into wire-like structures, origami structures, photonic crystal-like assemblies, liquid-crystal phases, and thin films. These structures can be made to serve as scaffolds for the organization of various organic molecules and nanoparticles. The properties of nanostructures can be modified by the use of DNA and DNA modified by the surfactants. $\odot 2012$ Society of Photo-Optical Instrumentation Engineers (SPIE). [DOI: 10.1117/1.JNP.6.064505]
\end{abstract}

Keywords: DNA self-assembly; DNA liquid crystals; nanoparticles ordering; DNA wires; DNA nanophotonics; DNA nanocrystals; DNA-origami; DNA-CTMA complex.

Paper 12025V received Mar. 8, 2012; revised manuscript received Jun. 8, 2012; accepted for publication Jun. 8, 2012; published online Sep. 4, 2012.

\section{Introduction}

The deoxyribonucleic acid (DNA) molecule is well known as a blueprint of life, amazingly rich in information content and very robust. However, its unique structural features and powerful recognition capabilities can also be of interest for assembling artificial structures for a variety of applications. This review mostly concentrates on the perspectives of use of DNA in nanophotonics: the technology of generating and manipulating light quanta on the nanoscale. A DNA helix is itself a nanoobject that can be manipulated in various ways, but it can also be treated as a versatile molecular scaffold for building nanoscale devices from the bottom up. ${ }^{1}$ DNA's unique properties are intensively studied from different points of view: as a molecular wire, as a drug delivery system, a ladder for ordered arrangements of various nanostructures, a spacer to control distances between nanoobjects, etc.

In this article, we review the use of DNA in the material science approaches, mainly through DNA's ability to provide order to molecules of dyes and/or nanoparticles (NPs) that are attached or interacting with the DNA helices. We describe the potential for a single DNA helix to behave as a robust building block of nanoscale systems, and we also discuss the ordering of DNA and light-responsive elements on a macroscopic scale, where liquid crystalline properties of DNA play an important role. Finally, we present how a modification of DNA, soluble in organic solvents, can be incorporated into optoelectronic and nanophotonic devices.

\section{DNA Helix-Ordering of Molecules and Nanoparticles for Applications in Nanophotonics}

\subsection{DNA as a Molecular Wire}

The elongated shape of the DNA molecule suggests that it is a wire capable of transmitting signals along its length. On the one hand, DNA is a rather poor electrical conductor, and its application in molecular electronics will be difficult. On the other hand, DNA possesses well-defined binding sites, where various molecules, from small dyes to large and complex

0091-3286/2012/\$25.00 ㄷ 2012 SPIE 
macromolecules, can be precisely located. Biochemists and genetic engineers have developed advanced techniques for the manipulation of DNA chains and multiple chemistry procedures of well-controlled DNA labeling. These robust possibilities of organization of organic markers on the DNA scaffold can be easily adapted for organization of photoactive molecules. Su et al. pointed out some of the latest achievements in the field of energy transfer via dyes organized on single DNA strands and on double-stranded DNA. ${ }^{2}$ Unidirectional energy transfer over the length of $40 \mathrm{bp}(\sim 13 \mathrm{~nm})$ on one DNA chain was observed with the efficiency of $20 \%$. $^{3}$ In order to achieve better efficiency, a template DNA strand, biotinylated at one end and labeled with injector (Rhodamine G) at the other end, was immobilized on a streptavidin-coated surface. ${ }^{4}$ Short complementary strands were doped with transmitting chromophores (tetramethylrhodamine, ATTO dyes, etc.) and the emitting dye (Cy5.5) and hybridized to the template. This five-color system demonstrated an energy transfer efficiency of $85 \%$. Apart from the hybridized dye-labeled short oligonucleotides, DNA stained with intercalators of groove-binders exhibits efficient energy transfer and can be considered to be photonic molecular wires. Such structures were expanded to two ${ }^{5}$ and three dimensions [see Fig. 1(a)]. ${ }^{6}$

Shionoya, Muller, and others introduced a concept of combining DNA and functional metal complexes into one molecular wire. ${ }^{10,11}$ More recently, single-molecule break junctions of metallo-DNA with single wall carbon nanotubes as point contacts were applied for charge transport. Incorporation of chelating agents enables the switching of conductive properties and opens up the possibility to build single-molecule devices, such as molecular switches or sensors. ${ }^{12}$

DNA electric properties are still a question being explored and debated, as various reports describe DNA as insulator, semiconductor, or conductor. ${ }^{13,14}$ It has been shown in the paper by Pawlicka at al. that the conductivity can by improved by adding the plasticizer such as poly(3,4-ethylenedioxythiophene)/poly(styrenesulfonate) (PEDOT:PSS), poly(o-ethoxyaniline) (POEA) or electrochromic dye Prussian Blue (PB). ${ }^{15}$ However, the DNA structure itself can serve as a reproducible scaffold where photoactive molecules can be positioned with nanometric precision.

\subsection{DNA as a Template for Synthesis of Nanoparticles}

The predictable order exhibited by DNA helices and their chemical stability make them attractive as agents used for controlling the synthesis of various NPs. Both single-strand DNA (ssDNA) and double-strand DNA (dsDNA) have been proved to be effective templates for the synthesis and ordering of nanometric objects. ${ }^{16}$ Noble metal (plasmonic) NPs are an example of a class of nanoobjects that can be successfully grown onto DNA templates. Standard synthesis of such

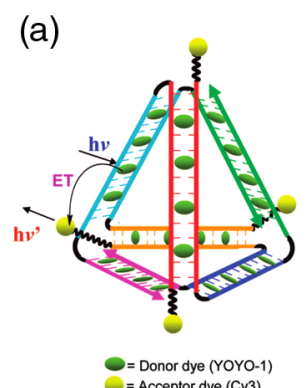
$=$ Acceptor dye (Cy3) (b)

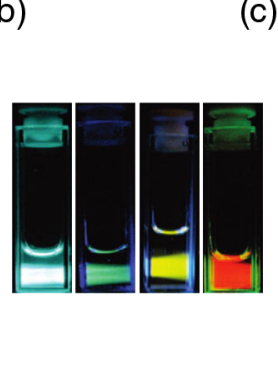

(c)

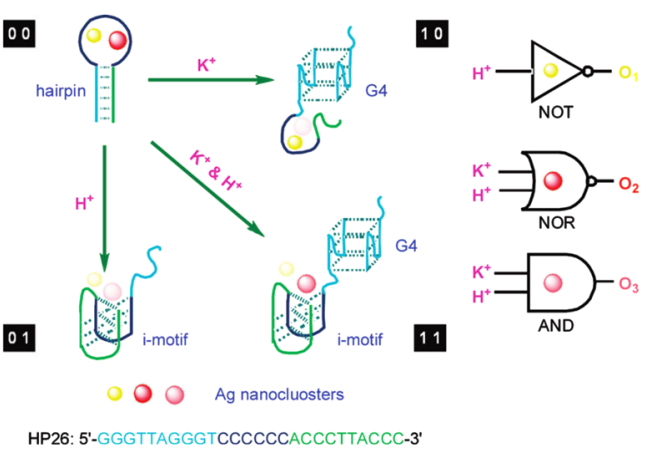

Fig. 1 (a) Schematic energy transfer (ET) in a tetrahedron DNA nanotag loaded with YOYO-1 intercalated dyes and covalently attached Cy3 acceptor dyes. ${ }^{5}$ (b) Pictures of emissive solutions of ss-DNA-encapsulated Ag nanoclusters created in: 5'-CCCTTTAACCCC-3' (blue), 5'CCCTCTTAACCC-3' (green), 5'-CCCTTAATCCCC-3' (yellow), 5'-CCTCCTTCCTCC-3' (red). (c) Schematic diagram of logic operations based on HP26-tuned fluorescent Ag nanoclusters. (Right) Corresponding symbols of logic gates. $\mathrm{K}^{+}$and $\mathrm{H}^{+}$serve as two inputs to trigger the allosterism of HP26 and modulate the fluorescence output. ${ }^{7,8,9}$ 
NPs involves the reduction of metal ions with reducing agents (e.g., citrate or borohydride). As an example of the advantage of performing such reactions in the presence of DNA, reducing $\mathrm{Ag}^{+}$ions in the presence of additional cytosine bases of short ssDNA was shown to lead to formation of NIR-emitting, water-soluble Ag nanoclusters. ${ }^{7}$ These few atom clusters possess high photostability, very good dispersibility in water, and very high single-molecule emission rates with strong antibunching. All these features yield great promise for the application of DNA-capped nanoclusters, introduced both in vitro and in vivo, in single-molecule imaging. The influence of the sequence, length, and arrangement of ssDNA on nanoparticle properties has been investigated. ${ }^{8,17}$ Modifications of the sequence resulted in the tuning of excitation and emission spectra of nanocrystals [Fig. 1(b)], with the photostability of DNA-NP complexes increasing with the increase of the emission wavelength. Moreover, when a DNA sequence producing a secondary intramolecular i-motif structure was introduced in Ag nanocluster formation, strong red luminescence was observed at $\mathrm{pH} 6$, giving way to green luminescence at $\mathrm{pH} 8$ to $9 .{ }^{17}$ Multiple logic operations can also be performed, evoked by changes in the concentrations of $\mathrm{K}^{+}$and $\mathrm{H}^{+}$ions, which in turn modify the structure of DNA. ${ }^{9}$

The presence of DNA molecules with various sequences during the nanoparticle synthesis determines their morphology. Gold NPs synthesized with the addition of poly(A), poly(C), and poly $(\mathrm{G})$ adopt a flower like shape, whereas poly(T) results in a spherical shape ${ }^{18}$ Synthesis in the presence of short ssDNA oligonucleotides (1 to 12 nucleotides) was also performed, ${ }^{19}$ and the homogeneity of nanoparticle size and shape (as well as stability in salt solutions) was found to increase with the length of the oligonucleotides. These DNA-mediated changes of nanoparticle morphology allow one to easily modify the optical properties, as well as influence the cellular uptake and biocompatibility of gold NPs. It appears that tuning of optical properties in nanoclusters using DNA oligonucleotides may be a simple and efficient technique compared to adjusting the optical properties of fluorescent dyes or of larger NPs. An interesting feature of DNA-tuned $\mathrm{Ag}$ nanoclusters is that they can also work as molecular logic gates exhibiting multichannel fluorescence output [Fig. 1(c)].

Interestingly, the chiral nature of the DNA helix may give rise to induced optical activity of the NPs synthesized on DNA templates. Chiral silver and gold nanoclusters ${ }^{20}$ and $\mathrm{NPs}^{21}$ have been prepared. The chirality of metal nanoclusters originates mainly from a high probability of packing $\mathrm{Ag}$ and $\mathrm{Au}$ atoms into low-symmetry chiral configurations, as was confirmed with theoretical computations [Fig. 2(a)]. ${ }^{22,23}$ On the other hand, larger NPs exhibit circular dichroism, most probably due to their chiral organization on DNA helix [Fig. 2(b)]. Several review articles describing DNA and other chiral molecules inducing chirality in metal NPs have been published recently. ${ }^{23-27}$ They demonstrate the increasingly growing interest in optically active nanostructures and their potential applications in chemical synthesis, biosensing, drug delivery, production of optical components, chiral negative index materials, and nanophotonic devices.

DNA has the ability to control the growth and morphology of quantum dots (QDs) as well. CdTe nanocrystals were synthesized in a one-pot procedure with a bifunctional DNA strand. ${ }^{29}$ One part of DNA, with a phosphorothioate end, served as a ligand binding to the QD surface, whereas the other part of DNA was a molecular recognition element. Differences in structural and optical properties of CdTe QD capped with A, G, T, or C oligonucleotides of various number of nucleotides were investigated. ${ }^{30}$ Stable and highly luminescent DNA-labeled QDs were then assembled in a well-controlled manner. QD complexes of up to three different sizes of nanocrystals were constructed. Their optical properties were dependent on the energy transfer between incorporated QDs and could be controlled by external stimuli (e.g., pH). Syntheses of DNA-capped cadmium and lead chalcogenide-based nanocrystals were also presented in the literature, ${ }^{31}$ and a strong influence of DNA on morphology and optical properties of the products was confirmed.

\subsection{Ordering and Assembling of Gold NPs with DNA Strands}

Thiol-functionalized DNA can be easily attached to gold surfaces and thus made to serve as a perfect link between gold NPs and nanoclusters, with full reversibility of binding of linking 
(a)
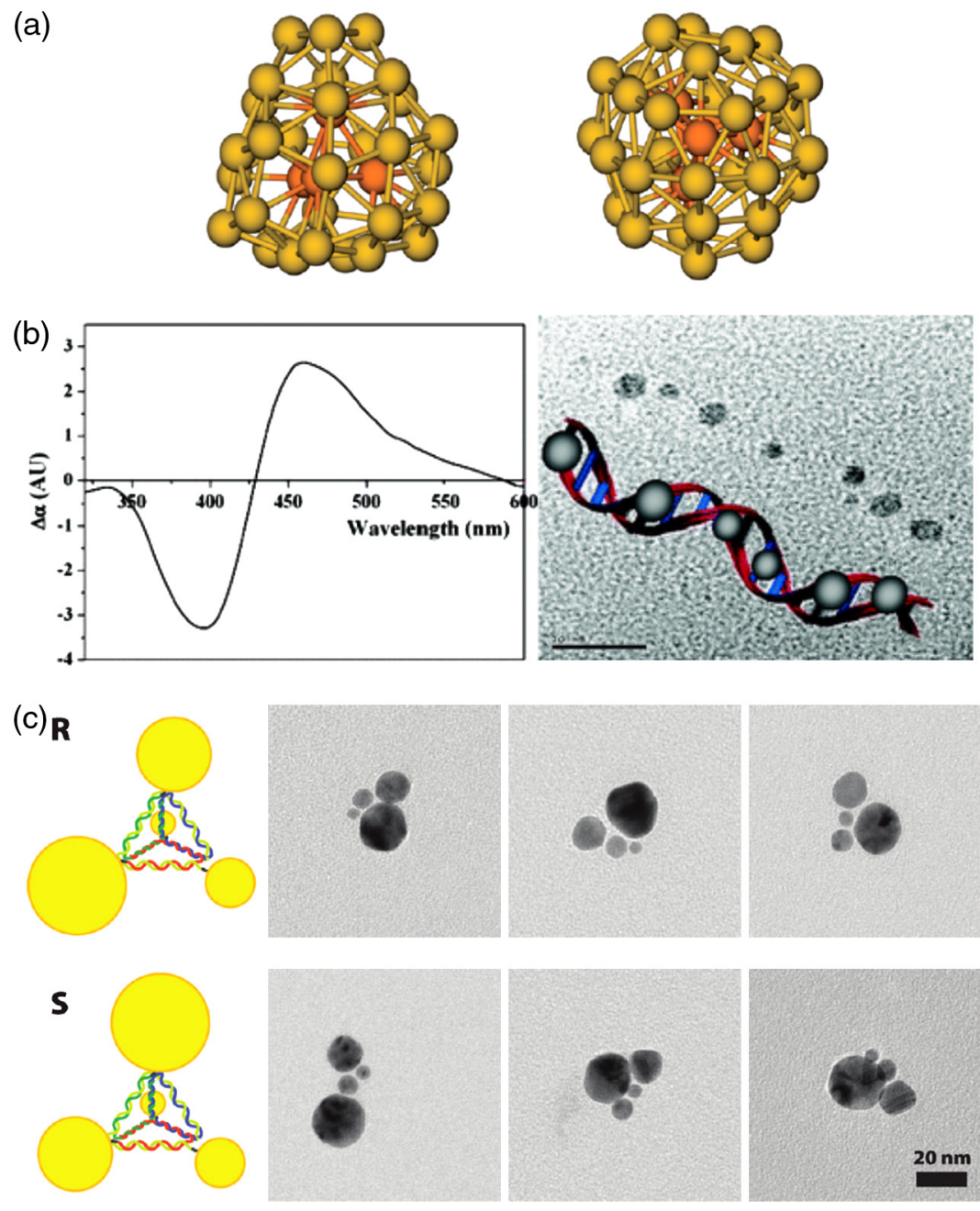

Fig. 2 (a) Selected energy-minimum chiral structures found for the $A_{34}$ cluster using DFT calculations. $^{22}$ (b) A circular dichroism spectrum of silver nanocrystals grown on a poly(dG)poly $(\mathrm{dC})$ dsDNA scaffold. ${ }^{21}$ (c) Examples of chiral pyramids of gold NPs linked with DNA. Top row, R; bottom row, S. ${ }^{28}$

chains. High specificity of the binding comes from the hybridization of two complementary ssDNAs on two different NPs or hybridization of these strands through a duplex-bridge DNA. Numerous review papers and monographs have been recently published on this broad subject. ${ }^{32-36}$ The linking can lead to the formation of various size plasmonic molecules (dimers, trimers, etc.), with NPs of the same or different sizes $;{ }^{37-39}$ furthermore, one can obtain regularly spaced plasmonic nanoparticle chains in polymerization-like reactions. ${ }^{40}$ Finally, threedimensional ordered arrays of gold NPs can be created ${ }^{41,42}$ with advanced procedures of the synthesis of well-defined plasmonic super-crystals, which have been recently described in Macfarlane et al. ${ }^{43}$ Among various geometries, chiral species were demonstrated, with gold NPs of four sizes, assembled with DNA linkers into chiral pyramides [Fig. 2(c)]. ${ }^{28}$ Many of the structures presented can successfully incorporate various lengths of the DNA strands and sizes of metal NPs, as well as inorganic nanocrystals. Changes of the geometry and relative positions of these building blocks have been shown to induce modifications of the optical properties. As a result, multifunctional, tunable and environmentally-sensitive nanostructures can be obtained. They are finding various interesting applications in nanophotonic systems (e.g., plasmon-based nanolenses). ${ }^{44}$

Not only can the structural properties of DNA be exploited in nanotechnology, but also the DNA synthesis techniques, developed by biologists and genetic engineers, may be adopted there 
(a)
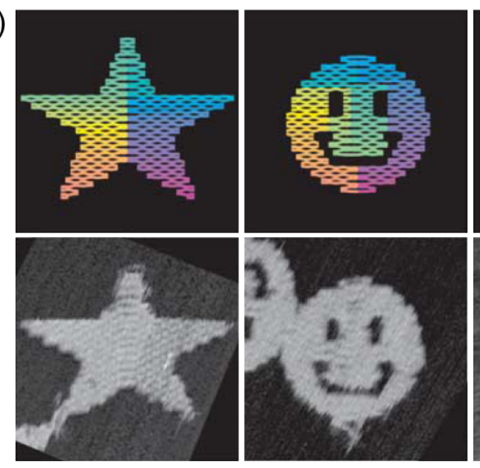

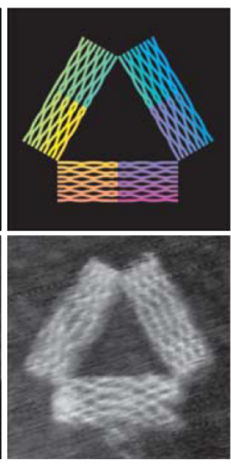

(b)

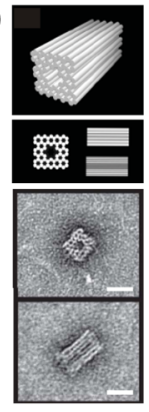

(c)

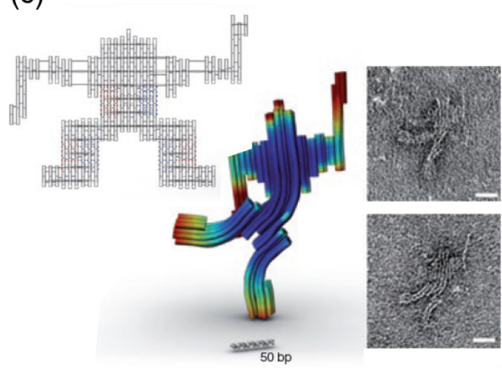

(d)

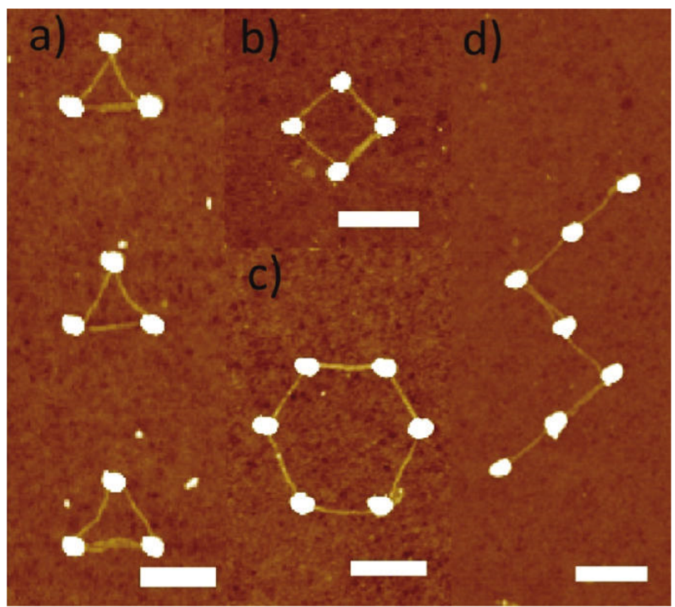

Fig. 3 (a) DNA-origami-top row, diagrams showing the bend of helices (color indicates the basepair index along the folding path; red-1st base, purple-7000th. Bottom row, AFM images, size $165 \times 165 \mathrm{~nm}^{2}{ }^{48}$ (b) Three-dimensional DNA-origami schemes and transmission electron microscopy (TEM) images of perspective and projection views of hollow-cylinder scale bars at $20 \mathrm{~nm} .{ }^{51}$ (c) Left-CanDo-designed multilayer DNA-origami object; middle-CanDo three-dimensional structure and local flexibility prediction shown as a heat map (local rms fluctuations, from blue to red); right-representative negative-stain TEM micrographs, scale bars $20 \mathrm{~nm}^{56}$ (d) Various structures formed by connecting gold islands with DNA-origami tubes: triangle (a); hexagon (b); square (c); $z$ shape (d). All scale bars are $300 \mathrm{~nm} .{ }^{54}$

as well. The polymerase chain reaction (PCR) is the standard DNA synthesis procedure, which offers exponential amplification of DNA strands identical with the template one. PCR, with DNA primers attached to gold and magnetic NPs, was performed, and nanoparticle aggregates were obtained. ${ }^{45}$ Then, PCR was applied to computer-controlled assembling of DNA-gold NP conjugates, with the size of the obtained nanostructures dependant on the number of PCR cycles. ${ }^{46}$ Interestingly, when the procedure is performed in a solution with a distribution of NP sizes, optically active dimers, trimers, tetramers, and pentamers are obtained. The product mixture is not racemic; it exhibits a strong positive circular dichroism signal whose origin has not yet been explained.

Diamond like lattices composed of gold NPs and viral particles, woven together and held in place by strands of DNA, were created ${ }^{47}$ Fabrication of such a structure-a distinctive mix of hard, metallic NPs and organic viral pieces known as capsids, linked by DNA—marks a big step toward creating a DNA-based photonic crystal that can manipulate visible light. The specific pieces of DNA were attached to gold NPs and viral particles, choosing the sequences and positioning them exactly to force the particles to arrange themselves into a crystal lattice that has a sodium thallide crystal structure. Such a self-assembled crystal lattice is potentially a central ingredient to a photonic crystal, which can be used for precise manipulation of light through blocking certain wavelengths of light while letting other colors pass. Applications for such 
DNA-base photonic crystal structures in fields like optical computing and telecommunications are foreseen, but manufacturing and durability remain serious challenges.

\subsection{DNA-Origami as a Multifunctional Nanoscaffold}

The unique property of complementary behavior of DNA bases became the basis of the controllable folding of DNA chains on a nanoscale. This method of manipulation of the chains, called DNA-origami, was introduced by Rothemund in $2006 .{ }^{48}$ It facilitates bending a single, long DNA strand with the aid of short staple strands with carefully designed sequences [Fig. 3(a)]. Since then, multiple periodically patterned two- and three-dimensional structures of DNA have been reported: twisted bundles of DNA, bent in a controllable way, ${ }^{49}$ threedimensional spherical shells and nanoflasks ${ }^{50}$ and three-dimensional monoliths and crosses made out of honeycomb lattices of DNA-origami ${ }^{51}$ [Fig. 3(b)]. Dietz and coworkers approached the automatic design and synthesis of DNA-origami structures by developing a computational tool (computer-aided engineering for DNA-origami: CanDo) for predicting the structure and providing information about the conditions under which DNA-origami objects can be expected to maintain their structure [Fig. 3(c)]. Recently, Zhao et al. presented a method to produce large area of DNA-origami by the superorigami or origami of origami method. ${ }^{52}$ Fabrication of largearea, mesoscopic arrays of gold NPs was made possible by depositing DNA-origami onto a lithographically patterned substrate, and the 5-nm gold NPs were then precisely bound to specific points on the DNA scaffold. ${ }^{53}$ The combination of top-down lithographies with bottom-up self-assembly was accomplished with DNA-origami nanotubes ${ }^{54}$ Due to appropriate functionalization with thiols, they formed connections between gold patterns produced with electron beam lithography [Fig. 3(d)]. Recently, the thermal stability of DNA constructs was improved by photo-cross-linking with 8-methoxypsoralen, ${ }^{55}$ which brings DNA-scaffolds closer to optoelectronic and nanophotonic applications.

DNA-origami-based elements of nanodevices have already been presented [e.g., nanoactuators $^{57}$ and tensegrity (tensional integrity) structures].$^{58}$ DNA-origami bundles were successfully used for controllable positioning of dye molecules in artificial light-harvesting antennas. ${ }^{59}$ DNA-scaffolds and DNA-nanoparticle assemblies find multiple applications in biodiagnostics and optical, electrochemical, or electrical biosensors. ${ }^{60,61}$ The combination of both of them may form sophisticated systems; e.g., a computational device with DNA nanomechanical self-assembled elements, which can divide a number by $3 .^{62}$ In this system, various ssDNAs serve as an input and the sequence of gold NPs bound to specifically ordered sticky ends of computed DNA sequence is an output signal.

Liu and coworkers examined the metallization of DNA-origami, as an enabling step toward the use of such DNA as templates for nanoelectronic circuits. ${ }^{63}$ DNA-origami makes it feasible to increase the complexity and flexibility needed for both the design and assembly of useful circuit templates. In addition, selective metallization of the DNA template is essential for circuit fabrication. Metallization of DNA-origami presents several challenges, including (1) the stability of the origami in the processes used for metallization, (2) the enhanced selectivity required to metallize small origami structures, (3) the increased difficulty of adhering small structures to the surface so that they will not be removed when subject to multiple metallization steps, and (4) the influence of excess staple strands present with the origami. The DNA templates were seeded with $\mathrm{Ag}$ and then plated with Au via an electroless deposition process. Both staple strand concentration and the concentration of ions in solution were found to have a significant impact. Selective continuous metal deposition was achieved, (height as small as $32 \mathrm{~nm}$ ), and the shape of branched origami was also retained after metallization. These results represent important progress toward the realization of DNA-templated nanocircuits. ${ }^{63}$ A novel method for producing complex metallic nanostructures of programmable design was presented. DNA-origami templates, modified to have DNA binding sites with a uniquely coded sequence, were adsorbed onto silicon dioxide substrates. Gold NPs, functionalized with the cDNA sequence, were then attached. These seed NPs were later enlarged, and even fused, by electroless deposition of silver. Using this method, a variety of metallic structures, including rings, pairs of bars, and $\mathrm{H}$ shapes, was constructed. ${ }^{64}$ 


\section{DNA as a Matrix for Ordering of Molecules and NPs}

\subsection{DNA Liquid Crystals}

The previous section described methods of ordering discrete DNA strands in two and three dimensions. A large-scale ordering of a high number of DNA chains is another opportunity for using DNA as a photonic material. In fact, the nature has already provided this behavior, and in aqueous solutions, DNA is known to exhibit liquid crystal (LC) ordering under certain conditions (lyotropic liquid crystallinity). LC phases of DNA were found in chromatin in cell nuclei ${ }^{65}$ bacteria carrying Blue Script plasmids, ${ }^{66,67}$ sperm heads, ${ }^{68}$ and capsids of viruses ${ }^{69}$ Depending on the temperature, DNA concentration and length, occurring counterions, and other polyions in the solution, DNA can be disorganized or can organize itself into a cholesteric, columnar hexagonal, or crystal phase. In the cholesteric phase, layers of DNA create a helical superstructure with interesting optical properties, such as selective reflection and transmission of circularly polarized light. ${ }^{70}$ DNA helices in the columnar hexagonal phase are packed parallel to one another, in a hexagonal structure. In both phases, various defects can occur, which can bend and deform DNA patterns. ${ }^{71}$ DNA-binding dyes follow the orientation of DNA in LC phases and can be perfect markers of DNA orientation. ${ }^{72}$ We have recently shown that, using a polarizationsensitive, two-photon fluorescence microscopy (ps-TPFM), we can map the exact position of dyes, and thus DNA, in three dimensions (Fig. 4). ${ }^{73}$ On the other hand, by controlling the ingredients of the functionalized DNA solution and external conditions, a specific LC phase can be obtained, with dyes ordered in a desirable manner. Not only standard DNA markers, but also photochromic dyes, were organized in such a system, ${ }^{74}$ proving the propensity to incorporate various functional molecules. LC phases are formed in sealed LC cells with appropriate conditions, but also in a simple system of drying droplets, where contact line pinning and radial forces produce a zigzag pattern [Fig. 4(a)]. ${ }^{72,75}$ This is an easy way of creating a millimeter-scale columnar phase of DNA and dye-doped DNA.
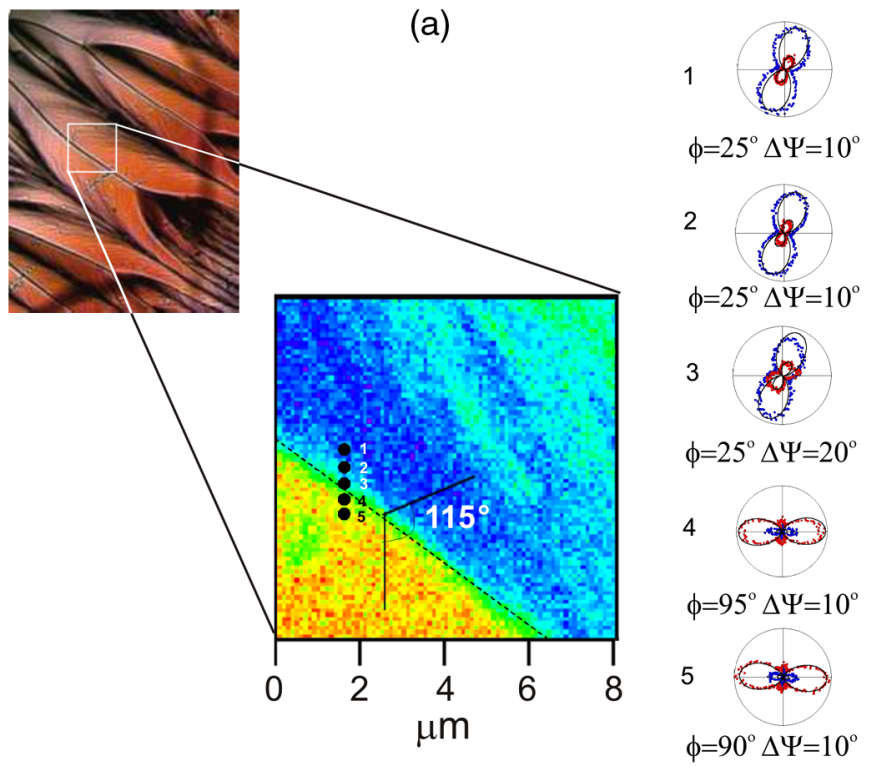

(b)

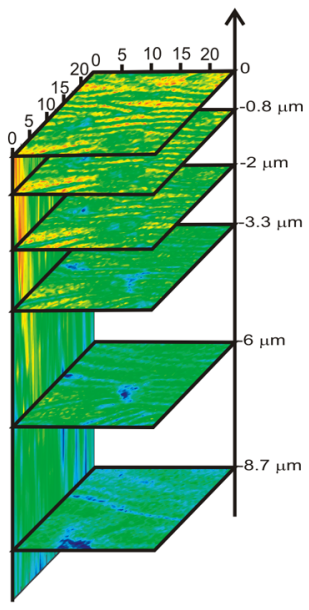

Fig. 4 (a) Polarized light microscopic (PLM) image of characteristic zigzag pattern at the perimeter of the dried DNA-dye solution drop and two-photon fluorescence intensity raster scan of the border region between two LC domains (white square in PLM image). Polar graphs (1 to 5) were measured in different points across the border line, and on that basis, the position of DNA in each domain was determined. ${ }^{72}$ (b) ps-TPFM scans at different depths of cholesteric DNA-dye phase and the $y, z$ cross-section of the sample. ${ }^{73}$ 
(a)

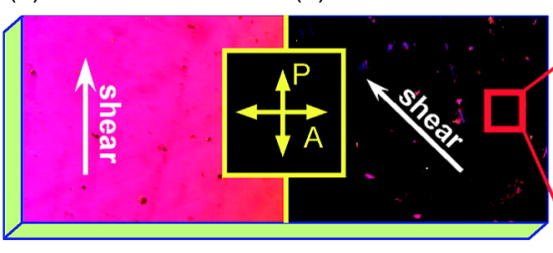

(b)

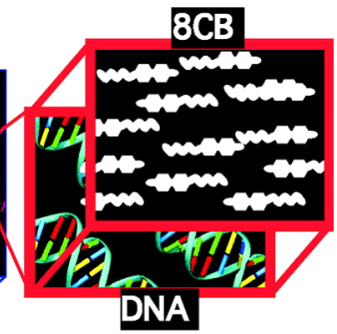

Fig. 5 Depolarized transmitted light microscope images between crossed polarizers of nematic 8CB between a sheared DNA film and a homeotropically aligning surface. (a) Pictures taken with polarization parallel to the shear direction indicate the uniform alignment of DNA, and the LC director pointing in the direction different from the direction of shear. (b) Rotation of the cell gives the extinction between the crossed polarizer and analyzer when the DNA shear direction is oriented at $50^{\circ}$ relative to the polarization direction. The inset presents the schematic orientation of $8 \mathrm{CB}$ molecules on DNA film. ${ }^{78}$

\subsection{DNA Thin Films}

DNA doped with the intercalating cyanine dyes were mixed with polyvinyl alcohol (PVA) and its alignment was studied. It has been proven that the DNA-PVA thin film is a highly anisotropic material that can be used, for example, for studies on structural properties for nucleic acids, but also its potential applications in photonics are envisaged. ${ }^{76}$ Thin layers of DNA/ Polyaniline/ $\mathrm{Ru}(\text { bpy })_{3}^{2+}$ complex and tris(8-hydroxyquinolinato)aluminum were combined in organic light-emitting diode (OLED) as the hole transport layer and the electron transport layer, respectively. ${ }^{77}$ Another approach is the exploitation of the DNA thin films for orientation of the LCs, such as 8CB and MBBA (see Fig. 5); the chirality of such systems has also been explored. $^{78}$

The mechanical properties of DNA-doped nanofiber hydrogels were shown in Shin et al. ${ }^{79}$ The elastic modulus of DNA is on the order of 0.3 to $1 \mathrm{GPa}$, similar to hard plastics, but at the same time, the molecule is extremely flexible, having the persistence length of $50 \mathrm{~nm} .{ }^{80}$

\section{Modified DNA Soluble in Organic Solvents-the Polymer Matrix for Optoelectronics}

DNA, as a biological material, is soluble in water. To introduce the solubility in organic solvents, substitution of sodium counter cations with cationic amphiphilic molecules is performed. Cationic lipids, ${ }^{81}$ hexadecyltrimethylammonium chloride (CTMA), ${ }^{82,83}$ and other surfactants are applied as ligands, binding to phosphate groups of DNA backbone. DNA-CTMA may also serve as a template for organizing dye molecules. Trapping of organic molecules between ligand molecules, or inside the DNA helix, was proposed in systems with fluorescent rhodamine G6 ${ }^{84}$ which then can be used for its lasing properties. Drying films of DNA-CTMA have selforganizing properties and very good processability for integration into large-area devices. ${ }^{82}$ Interestingly, the helical structure of DNA is preserved and can serve as a template for chiral organization of dopants. Circular dichroism of films of DNA-CTMA doped with hemicyanine NLO dye, 4[4-(dimethylamino)styryl]-1-docosylpyridinium (DMASDPB), was measured, and the full orientation structure along the chiral DNA double helices was confirmed. ${ }^{82}$ Chiral organization of molecules is an alternative for the polar organization required to produce second-harmonic generation (SHG). Dried films of DNA-CTMA, doped with the dye crystal violet, were SHG-active, without application of any external stimuli. ${ }^{85}$

The DNA-CTMA complex has been proposed as an ideal material for optoelectronics due to its unique electromagnetic and optical properties, which are summarized in Table $1 .^{86,87}$ Intrinsic nonlinear refractive index discourages to apply pure DNA as nonlinear optical material. ${ }^{88}$ However, DNA-CTMA has been applied as a cladding and host material in 
Table 1 Properties of the DNA-CTMA Complex..$^{86}$

Summary of Properties of DNA-CTMA

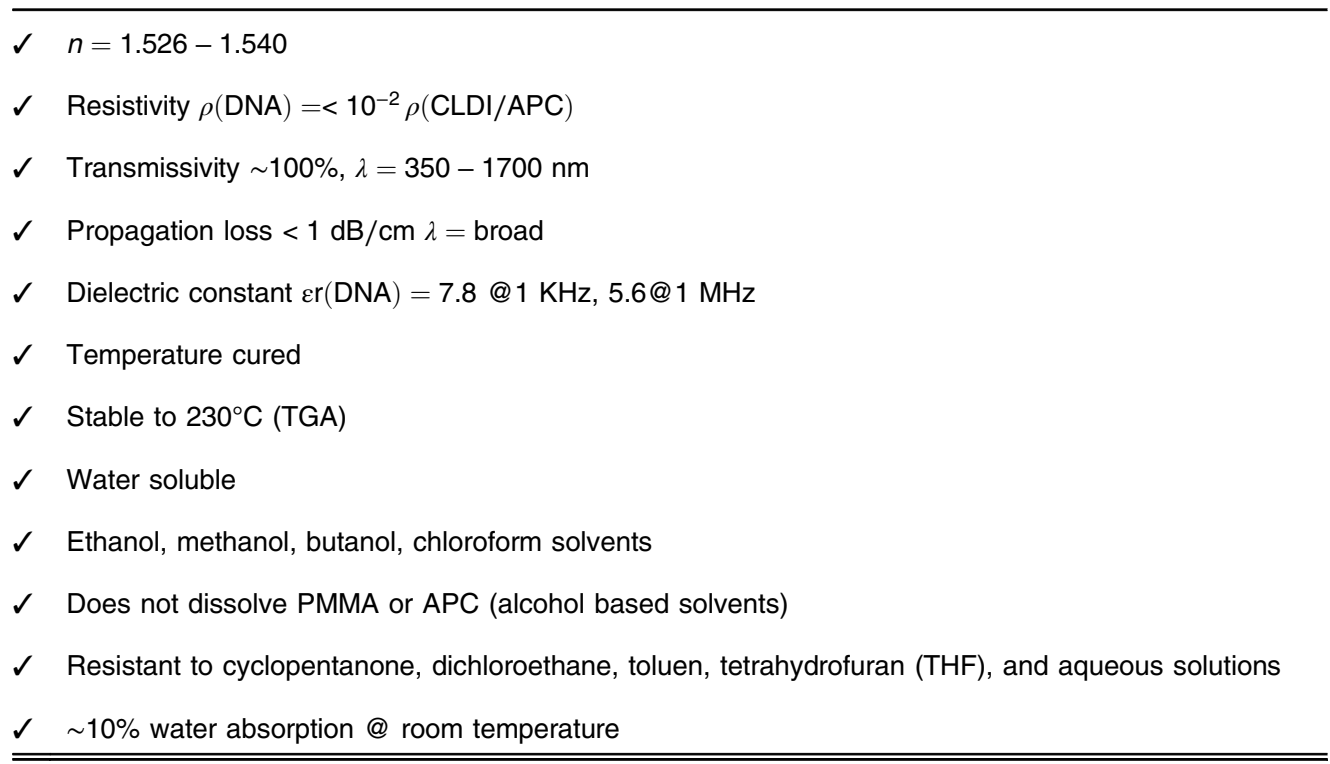

nonlinear optical devices. ${ }^{89}$ In organic field effect transistors, a thin layer of DNA-CTMA was introduced as gate dielectric, ${ }^{90}$ and in combination with quantum dots, it served as the efficient hole-transporting layer in light-emitting $\operatorname{diodes}^{91}$ and as an electron-blocking layer in OLEDs. ${ }^{92}$

Moreover, DNA-CTMA doped with photochromic dyes, such as Disperse Red 1, provided a favorable matrix for dynamic holography ${ }^{93,94}$ and other nonlinear optical features such as, for example, third-harmonic generation. ${ }^{95}$ DNA with cationic lipids containing TEMPO radicals exhibits a reversible two-stage charge/discharge processes with promising application as an organic radical battery,${ }^{96}$ whereas DNA loaded with the DMASDPB dye demonstrated improved photostability when compared to the PMMA matrix (to cite one example), which can be of importance for photonic applications (solid-state laser design). ${ }^{97}$ Amplified spontaneous emission was observed under nanosecond optical pumping, when the DNA-CTMA matrix was doped with rhodamine dye $\mathrm{e}^{98}$ or a combination of organic nonlinear optical dye and photochromic polymer. ${ }^{99}$ Recently, dielectric tunability in DNA-CTMA film at microwave frequencies was shown ${ }^{100}$ pointing out that the dominant polarization mechanism is ionic in nature and is caused by intentionally retaining excess ions in the DNA-CTMA precipitate during processing. Effective use of the DNA-CTMA matrix for fabrication of waveguides was presented in Zhou et al. ${ }^{101}$ The waveguides exhibited excellent single-mode output and high confinement of light; due to the sharp waveguide profile with very smooth surfaces and vertical sidewalls, low propagation losses were measured.

Any unfavorable characteristics of DNA-CTMA can be improved by formation of hybrid materials (e.g. DNA-ORMOCER nanocomposite). ${ }^{102}$ ORMOCER provides mechanical strength, and better hardness than the pure DNA-CTMA films and DNA serves as an efficient dye and nanoparticle-binding material.

\section{Conclusion and Perspectives}

With this review, we set out to convey that DNA is a very unique system, which has been studied for numerous applications, not only coming from its biological significance but also for material science applications. DNA's specific organization allows for the construction of photonic molecular systems, such as what is schematically shown in Fig. $6 .{ }^{103}$ 


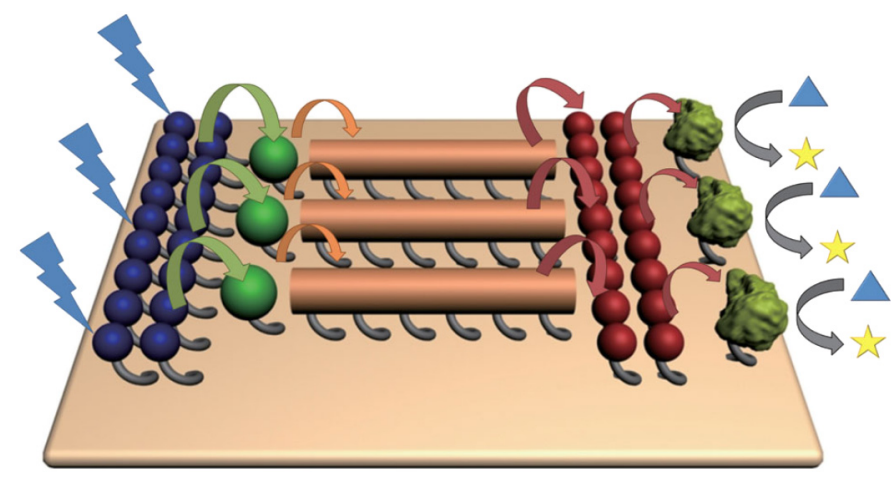

Fig. 6 A scheme of a DNA-based system for energy transfer and photonics. ${ }^{103}$

Light-harvesting systems can be arranged in a specific order, where the sequence of energy or charge transfer processes leads to optimum performance of channeling to create a new generation of photonic wires or conducting plasmonic devices (blue, green, and red balls and orange bars represent the photonic components that can serve as light-harvesting and energy-transfer materials). Other species can act as molecular sensing units by energy or electron accepting, where the light is converted to chemical potential (represented by the transformation of the substrate-triangles to the higher-energy product-stars).

Apart from the organization of photoactive species with single DNA helices, we would like to underline the ability of DNA to create LC phases, which can then be used as matrices for organization of other species, such as dyes or nanoobjects. The LC phases of DNA are undoubtedly of important biological importance (chromatin organization), but they also shall be explored for the positioning of the NPs and molecules in precisely chosen places. Then, the phase transition appearing in the LC DNA also can help the manipulation of the NPs, thus preparing a bistable system.

\section{Acknowledgments}

We are grateful to Professors M. Samoc and J. Sworakowski for their constructive comments and discussions and to Professor M. Buckle for biological inspirations. We acknowledge funding for this research from the Foundation for Polish Science, Program Welcome. This work was also supported by a statutory activity subsidy from the Polish Ministry of Science and Higher Education for the Faculty of Chemistry of Wroclaw University of Technology and National Science Centre Grant No. DEC-2011/01/N/ST5/02404.

\section{References}

1. Y. W. Kwon et al., "Materials science of DNA," J. Mater. Chem. 19, 1353-1380 (2009), http://dx.doi.org/10.1039/b808030e.

2. W. Su, V. Bonnard, and G. A. Burley, "DNA-Templated Photonic arrays and assemblies: design principles and future opportunities," Chem.-Eur. J. 17, 7982-7991 (2011), http://dx .doi.org/10.1002/chem.v17.29.

3. S. Vyawahare et al., "Nanometer-scale fluorescence resonance optical waveguides," Nano Lett. 4, 1035-1039 (2004), http://dx.doi.org/10.1021/nl049660i.

4. M. Heilemann et al., "Dissecting and reducing the heterogeneity of excited-state energy transport in DNA-based photonic wires," J. Am. Chem. Soc. 128, 16864-16875 (2006), http://dx.doi.org/10.1021/ja065585x.

5. H. Özhalıc1-Un̈al and B. A. Armitage, "Fluorescent DNA nanotags based on a selfassembled DNA tetrahedron," ACS Nano 3, 425-433 (2009), http://dx.doi.org/10.1021/ nn800727x.

6. A. L. Benvin et al., "Fluorescent DNA nanotags: Supramolecular fluorescent labels based on intercalating dye arrays assembled on nanostructured DNA templates," J. Am. Chem. Soc. 129, 2025-2034 (2007), http://dx.doi.org/10.1021/ja066354t. 
7. T. Vosch et al., "Strongly emissive individual DNA-encapsulated Ag nanoclusters as single-molecule fluorophores," Proc. Nat. Acad. Sci. 104, 12616-12621 (2007), http:// dx.doi.org/10.1073/pnas.0610677104.

8. C. I. Richards et al., "Oligonucleotide-stabilized Ag nanocluster fluorophores," J. Am. Chem. Soc. 130, 5038-5039 (2008), http://dx.doi.org/10.1021/ja8005644.

9. T. Li et al., "Ion-tuned DNA/Ag fluorescent nanoclusters as versatile logic device," ACS Nano 5, 6334-6338 (2011), http://dx.doi.org/10.1021/nn201407h.

10. J. Muller, "Chemistry: metals line up for DNA," Nature 444, 698-698 (2006), http://dx.doi .org/10.1038/444698a.

11. G. H. Clever and M. Shionoya, "Metal-base pairing in DNA," Coord. Chem. Rev. 254, 2391-2402 (2010), http://dx.doi.org/10.1016/j.ccr.2010.04.014.

12. S. Liu et al., "Direct conductance measurement of individual metallo-DNA duplexes within single-molecule break junctions," Angew. Chem. Int. Edit. 50, 8886-8890 (2011), http://dx.doi.org/10.1002/anie.v50.38.

13. M. Taniguchi and T. Kawai, "DNA electronics," Physica E-Low-Dimens. Sys. Nanostruct. 33(1), 1-12 (2006), http://dx.doi.org/10.1016/j.physe.2006.01.005.

14. X. Guo et al., "Conductivity of a single DNA duplex bridging a carbon nanotube gap," Nat. Nanotechnol. 3, 163-167 (2008), http://dx.doi.org/10.1038/nnano.2008.4.

15. A. Pawlicka et al., "Ionically conducting DNA-based membranes for eletrochromic devices," Syn. Metals 161, 2329-2334 (2011), http://dx.doi.org/10.1016/j.synthmet 2011.08.043.

16. L. Berti and G. A. Burley, "Nucleic acid and nucleotide-mediated synthesis of inorganic nanoparticles," Nat Nano 3, 81-87 (2008), http://dx.doi.org/10.1038/nnano.2007.460.

17. B. Sengupta et al., "DNA templates for fluorescent silver clusters and i-motif folding," J. Phys. Chem. C 113, 19518-19524 (2009), http://dx.doi.org/10.1021/jp906522u.

18. Z. D. Wang et al., "DNA-mediated control of metal nanoparticle shape: one-pot synthesis and cellular uptake of highly stable and functional gold nanoflowers," Nano Lett. 10, 1886-1891 (2010), http://dx.doi.org/10.1021/n1100675p.

19. L. G. Xu et al., "New synthesis strategy for DNA functional gold nanoparticles," J. Phys. Chem. C 115, 3243-3249 (2011), http://dx.doi.org/10.1021/jp109688q.

20. J. T. Petty et al., "DNA-templated Ag nanocluster formation," J. Am. Chem. Soc. 126, 5207-5212 (2004), http://dx.doi.org/10.1021/ja031931o.

21. G. Shemer et al., "Chirality of silver nanoparticles synthesized on DNA," J. Am. Chem. Soc. 128, 11006-11007 (2006), http://dx.doi.org/10.1021/ja063702i.

22. A. Lechtken et al., "Au34-: a chiral gold cluster?" Angew. Chem. Int. Edit. 46, 2944-2948 (2007), http://dx.doi.org/10.1002/(ISSN)1521-3773.

23. C. Noguez and I. L. Garzon, "Optically active metal nanoparticles," Chem. Soc. Rev. 38, 757-771 (2009), http://dx.doi.org/10.1039/b800404h.

24. C. Gautier and T. Bürgi, "Chiral gold nanoparticles," ChemPhysChem 10, 483-492 (2009), http://dx.doi.org/10.1002/cphc.v10:3.

25. A. Guerrero-Martínez et al., "From individual to collective chirality in metal nanoparticles," Nano Today 6, 381-400 (2011), http://dx.doi.org/10.1016/j.nantod.2011.06.003.

26. V. Kitaev, "Chiral nanoscale building blocks-from understanding to applications," J. Mater. Chem. 18, 4745-4749 (2008), http://dx.doi.org/10.1039/b808054b.

27. A. O. Govorov et al., "Chiral nanoparticle assemblies: circular dichroism, plasmonic interactions, and exciton effects," J. Mater. Chem. 21, 16806-16818 (2011), http://dx .doi.org/10.1039/c1jm12345a.

28. A. J. Mastroianni, S. A. Claridge, and A. P. Alivisatos, "Pyramidal and chiral groupings of gold nanocrystals assembled using DNA scaffolds," J. Am. Chem. Soc. 131, 8455-8459 (2009), http://dx.doi.org/10.1021/ja808570g.

29. N. Ma, E. H. Sargent, and S. O. Kelley, "One-step DNA-programmed growth of luminescent and biofunctionalized nanocrystals," Nat. Nanotechnol. 4, 121-125 (2009), http://dx .doi.org/10.1038/nnano.2008.373.

30. G. Tikhomirov et al., "DNA-based programming of quantum dot valency, self-assembly, and luminescence," Nat. Nanotechnol. 6, 485-490 (2011), http://dx.doi.org/10.1038/ nnano.2011.100. 
31. N. Ma, G. Tikhomirov, and S. O. Kelley, "Nucleic Acid-passivated semiconductor nanocrystals: biomolecular templating of form and function," Acc. Chem. Res. 43, 173-180 (2010), http://dx.doi.org/10.1021/ar900046n.

32. S. J. Tan et al., "Building plasmonic nanostructures with DNA," Nat. Nanotechnol. 6, 268-276 (2011), http://dx.doi.org/10.1038/nnano.2011.49.

33. F. Westerlund and T. Bjornholm, "Directed assembly of gold nanoparticles," Curr. Opin. Colloid in. Sci. 14, 126-134 (2009), http://dx.doi.org/10.1016/j.cocis.2008.07.002.

34. Z. Yuanqin and D. Zhaoxiang, "Nanostructures and nanomaterials via DNA-based self-assembly" in Materials Science of DNA, 1st Ed.,CRC Press, pp. 13-48 (2011).

35. M. R. Jones et al., "Templated techniques for the synthesis and assembly of plasmonic nanostructures," Chem. Rev. 111, 3736-3827 (2011), http://dx.doi.org/10.1021/ cr1004452.

36. O. I. Wilner and I. Willner, "Functionalized DNA nanostructures," Chem. Rev. 112, 2528-2556 (2012), http://dx.doi.org/10.1021/cr200104q.

37. X. Xu et al., "Asymmetric functionalization of gold nanoparticles with oligonucleotides," J. Am. Chem. Soc. 128, 9286-9287 (2006), http://dx.doi.org/10.1021/ja061980b.

38. M. M. Maye et al., "Stepwise surface encoding for high-throughput assembly of nanoclusters," Nat. Mater. 8, 388-391 (2009), http://dx.doi.org/10.1038/nmat2421.

39. M. P. Busson et al., "Optical and topological characterization of gold nanoparticle dimers linked by a single DNA double strand," Nano Lett. 11, 5060-5065 (2011), http://dx.doi .org/10.1021/nl2032052.

40. Z. X. Deng et al., "DNA-encoded self-assembly of gold nanoparticles into onedimensional arrays," Angew. Chem. Int. Edit. 44, 3582-3585 (2005), http://dx.doi.org/ 10.1002/(ISSN)1521-3773.

41. D. Nykypanchuk et al., "DNA-guided crystallization of colloidal nanoparticles," Nature 451, 549-552 (2008), http://dx.doi.org/10.1038/nature06560.

42. S. Y. Park et al., "DNA-programmable nanoparticle crystallization," Nature 451, 553-556 (2008), http://dx.doi.org/10.1038/nature06508.

43. R. J. Macfarlane et al., "Nanoparticle superlattice engineering with DNA," Science 334, 204-208 (2011), http://dx.doi.org/10.1126/science.1210493.

44. S. Bidault, F. J. García de Abajo, and A. Polman, "Plasmon-based nanolenses assembled on a well-defined DNA template," J. Am. Chem. Soc. 130, 2750-2751 (2008), http://dx.doi .org/10.1021/ja711074n.

45. H. B. Shen et al., "Polymerase chain reaction of nanoparticle-bound primers," Biophys. Chem. 115, 63-66 (2005), http://dx.doi.org/10.1016/j.bpc.2005.01.001.

46. W. Chen et al., "Nanoparticle superstructures made by polymerase chain reaction: collective interactions of nanoparticles and a new principle for chiral materials," Nano Lett. 9, 2153-2159 (2009), http://dx.doi.org/10.1021/n1900726s.

47. P. Cigler et al., "DNA-controlled assembly of a NaTl lattice structure from gold nanoparticles and protein nanoparticles," Nat. Mater. 9, 918-922 (2010), http://dx.doi.org/10 .1038/nmat2877.

48. P. W. K. Rothemund, "Folding DNA to create nanoscale shapes and patterns," Nature 440, 297-302 (2006), http://dx.doi.org/10.1038/nature04586.

49. H. Dietz, S. M. Douglas, and W. M. Shih, "Folding DNA into twisted and curved nanoscale shapes," Science 325, 725-730 (2009), http://dx.doi.org/10.1126/science .1174251 .

50. D. Han et al., "DNA origami with complex curvatures in three-dimensional space," Science 332, 342-346 (2011), http://dx.doi.org/10.1126/science.1202998.

51. S. M. Douglas et al., "Self-assembly of DNA into nanoscale three-dimensional shapes," Nature 459, 414-418 (2009), http://dx.doi.org/10.1038/nature08016.

52. Z. Zhao, Y. Liu, and H. Yan, "Organizing DNA origami tiles into larger structures using preformed scaffold frames," Nano Lett. 11, 2997-3002 (2011), http://dx.doi.org/10.1021/ nl201603a.

53. A. M. Hung et al., "Large-area spatially ordered arrays of gold nanoparticles directed by lithographically confined DNA origami," Nat. Nanotechnol. 5, 121-126 (2010), http://dx .doi.org/10.1038/nnano.2009.450. 
54. B. Ding et al., "Interconnecting gold islands with DNA origami nanotubes," Nano Lett. 10, 5065-5069 (2010), http://dx.doi.org/10.1021/nl1033073.

55. A. Rajendran et al., "Photo-cross-linking-assisted thermal stability of DNA origami structures and its application for higher-temperature self-assembly," J. Am. Chem. Soc. 133, 14488-14491 (2011), http://dx.doi.org/10.1021/ja204546h.

56. C. E. Castro et al., "A primer to scaffolded DNA origami," Nat. Meth. 8, 221-229 (2011), http://dx.doi.org/10.1038/nmeth.1570.

57. M. Marini et al., "A revertible, autonomous, self-assembled DNA-origami nanoactuator," Nano Lett. 11, 5449-5454 (2011), http://dx.doi.org/10.1021/nl203217m.

58. T. Liedl et al., "Self-assembly of three-dimensional prestressed tensegrity structures from DNA," Nat. Nanotechnol. 5, 520-524 (2010), http://dx.doi.org/10.1038/nnano .2010 .107 .

59. P. K. Dutta et al., "DNA-directed artificial light-harvesting antenna," J. Am. Chem. Soc. 133, 11985-11993 (2011), http://dx.doi.org/10.1021/ja1115138.

60. N. L. Rosi and C. A. Mirkin, "Nanostructures in biodiagnostics," Chem. Rev. 105, 1547-1562 (2005), http://dx.doi.org/10.1021/cr030067f.

61. H. Wang et al., "Nucleic acid conjugated nanomaterials for enhanced molecular recognition," ACS Nano 3, 2451-2460 (2009), http://dx.doi.org/10.1021/nn9006303.

62. B. Chakraborty, N. Jonoska, and N. C. Seeman, "A programmable transducer selfassembled from DNA," Chem. Sci. 3, 168-176 (2012), http://dx.doi.org/10.1039/ c1sc00523e.

63. J. Liu et al., "Metallization of branched DNA origami for nanoelectronic circuit fabrication," ACS Nano 5, 2240-2247 (2011), http://dx.doi.org/10.1021/nn1035075.

64. M. Pilo-Pais et al., "Connecting the nanodots: programmable nanofabrication of fused metal shapes on DNA templates," Nano Lett. 11, 3489-3492 (2011), http://dx.doi.org/ $10.1021 / \mathrm{nl} 202066 \mathrm{c}$.

65. F. Livolant, "Cholesteric organization of DNA invivo and invitro," Eur. J. Cell Biol. 33, 300-311 (1984).

66. Z. Reich, E. Wachtel, and A. Minsky, "Liquid-crystalline mesophases of plasmid DNA in bacteria," Science 264, 1460-1463 (1994), http://dx.doi.org/10.1126/science.8197460.

67. P. Hanczyc et al., "Spontaneous formation of liquid crystalline phases and phase transitions in highly concentrated plasmid DNA," Liq. Cryst. 38, 461-468 (2011), http://dx.doi .org/10.1080/02678292.2011.552740.

68. F. Livolant, "Cholesteric organization of DNA in the stallion sperm head," Tissue and Cell 16, 535-555 (1984), http://dx.doi.org/10.1016/0040-8166(84)90029-6.

69. A. Leforestier and F. Livolant, "The bacteriophage genome undergoes a succession of intracapsid phase transitions upon DNA ejection," J. Mol. Biol. 396, 384-395 (2010), http://dx.doi.org/10.1016/j.jmb.2009.11.047.

70. A. Leforestier and F. Livolant, "Supramolecular ordering of DNA in the cholesteric liquid crystalline phase: an ultrastructural study," Biophys. J. 65, 56-72 (1993), http://dx.doi.org/ 10.1016/S0006-3495(93)81063-4.

71. F. Livolant and A. Leforestier, "Condensed phases of DNA: structures and phase transitions," Prog. Polym. Sci. 21, 1115-1164 (1996), http://dx.doi.org/10.1016/S0079-6700 (96)00016-0.

72. H. Mojzisova et al., "Polarization-sensitive two-photon microscopy study of the organization of liquid-crystalline DNA," Biophys. J. 97, 2348-2357 (2009), http://dx.doi.org/10 .1016/j.bpj.2009.07.053.

73. J. Olesiak-Banska et al., "Liquid crystal phases of DNA: evaluation of DNA organization by two-photon fluorescence microscopy and polarization analysis," Biopolymers 95, 365-375 (2011), http://dx.doi.org/10.1002/bip.v95.6.

74. J. Olesiak et al., "Liquid crystalline phases in DNA and dye-doped DNA solutions analysed by polarized linear and nonlinear microscopy and differential scanning calorimetry," Mater. Sci.-Poland 27, 813-823 (2009).

75. I. I. Smalyukh et al., "Structure and dynamics of liquid crystalline pattern formation in drying droplets of DNA," Phys. Rev. Lett. 96, 177801 (2006), http://dx.doi.org/10 .1103/PhysRevLett.96.177801. 
76. P. Hanczyc, B. Norden, and B. Åkerman, "DNA in a polyvinyl alcohol matrix and interactions with three intercalating cyanine dyes," J. Phys. Chem. B 115, 12192-12201 (2011), http://dx.doi.org/10.1021/jp205374t.

77. K. Nakamura et al., "Color-tunable multilayer organic light emitting diode composed of DNA complex and tris(8-hydroxyquinolinato)aluminum," Appl. Phys. Lett. 97, 193301 (2010), http://dx.doi.org/10.1063/1.3512861.

78. M. Nakata et al., "Liquid crystal alignment on a chiral surface: interfacial interaction with sheared DNA films," Langmuir 24, 10390-10394 (2008), http://dx.doi.org/10 $.1021 / 1 \mathrm{la} 800639 \mathrm{x}$.

79. M. K. Shin et al., "The effect of DNA on mechanical properties of nanofiber hydrogels," Appl. Phys. Lett. 93, 171903 (2008), http://dx.doi.org/10.1063/1.3009204.

80. K. Bloom, "Beyond the code: the mechanical properties of DNA as they relate to mitosis," Chromosoma 117, 103-110 (2008), http://dx.doi.org/10.1007/s00412-007-0138-0.

81. J. Q. Qu et al., "DNA-lipid complexes carrying carbazole and triphenylamine moieties: synthesis, and chiroptical and photoelectronic properties," Polymer 49, 3663-3670 (2008), http://dx.doi.org/10.1016/j.polymer.2008.06.039.

82. L. L. Wang, J. Yoshida, and N. Ogata, "Self-assembled supramolecular films derived from marine deoxyribonucleic acid (DNA)-cationic surfactant complexes: large-scale preparation and optical and thermal properties," Chem. Mater. 13, 1273-1281 (2001), http://dx .doi.org/10.1021/cm000869g.

83. E. M. Heckman et al., "Processing techniques for deoxyribonucleic acid: biopolymer for photonics applications," Appl. Phys. Lett. 87, 211115 (2005), http://dx.doi.org/10.1063/1 .2135205 .

84. Y. Kawabe et al., "Amplified spontaneous emission from fluorescent-dye-doped DNAsurfactant complex films," Adv. Mater. 12, 1281-1283 (2000), http://dx.doi.org/10 $.1002 /($ ISSN)1521-4095.

85. D. Wanapun et al., "DNA-based polymers as chiral templates for second-order nonlinear optical materials," ChemPhysChem 10, 2674-2678 (2009), http://dx.doi.org/10.1002/cphc .v10:15.

86. J. G. Grote et al., "DNA photonics [deoxyribonucleic acid]," Mol. Cryst. Liq. Cryst. 426, 3-17 (2005), http://dx.doi.org/10.1080/15421400590890615.

87. J. G. Grote, "Natural nanophotonics," Journal of Nanophotonics 2, 020301 (2008), http:// dx.doi.org/10.1117/1.2949248.

88. M. Samoc, A. Samoc, and J. G. Grote, "Complex nonlinear refractive index of DNA," Chem. Phys. Lett. 431, 132-134 (2006), http://dx.doi.org/10.1016/j.cplett.2006 .09.057.

89. E. M. Heckman et al., "DNA biopolymer conductive cladding for polymer electro-optic waveguide modulators," Appl. Phys. Lett. 98, 103304 (2011), http://dx.doi.org/10.1063/1 .3562953 .

90. P. Stadler et al., "Organic field-effect transistors and memory elements using deoxyribonucleic acid (DNA) gate dielectric," Org. Elec. 8, 648-654 (2007), http://dx.doi.org/10 .1016/j.orgel.2007.05.003.

91. Q. Sun et al., "Highly efficient quantum-dot light-emitting diodes with DNA-CTMA as a combined hole-transporting and electron-blocking layer," ACS Nano 3, 737-743 (2009), http://dx.doi.org/10.1021/nn8009079.

92. J. A. Hagen et al., "Enhanced emission efficiency in organic light-emitting diodes using deoxyribonucleic acid complex as an electron blocking layer," Appl. Phys. Lett. 88, 171109 (2006), http://dx.doi.org/10.1063/1.2197973.

93. A. Miniewicz et al., "Deoxyribonucleic acid-based photochromic material for fast dynamic holography," Appl. Phys. Lett. 91, 041118 (2007), http://dx.doi.org/10.1063/1 .2760169 .

94. B. Derkowska et al., "Influence of different peripheral substituents on the nonlinear optical properties of cobalt phthalocyanine core," J. Appl. Phys. 101, 083112 (2007), http://dx.doi .org/10.1063/1.2719281.

95. O. Krupka et al., "NLO properties of functionalized DNA thin films," Thin Solid Films 516, 8932-8936 (2008), http://dx.doi.org/10.1016/j.tsf.2007.11.089. 
96. J. Q. Qu et al., "Synthesis and properties of DNA complexes containing 2,2,6,6tetramethyl-1-piperidinoxy (TEMPO) moieties as organic radical battery materials," Chem.-Eur. J. 14, 3250-3259 (2008), http://dx.doi.org/10.1002/(ISSN)1521-3765.

97. Y. Ner et al., "Stabilization of fluorophore in DNA thin films," Appl. Phys. Lett. 95, 263701 (2009), http://dx.doi.org/10.1063/1.3262944.

98. Y. Kawabe et al., "Amplified spontaneous emission from fluorescent-dye-doped DNAsurfactant complex films," Adv. Mater. 12(17), 1281-1283 (2000), http://dx.doi.org/10 .1002/(ISSN)1521-4095.

99. L. Sznitko et al., "Biopolymer-based system doped with nonlinear optical dye as a medium for amplified spontaneous emission and lasing," Appl. Phys. Lett. 99, 031107 (2011), http://dx.doi.org/10.1063/1.3610566.

100. R. S. J. Aga et al., "Origin of dielectric tunability in DNA-CTMA film at microwave frequencies,” Proc. SPIE 8103, 81030L (2011), http://dx.doi.org/10.1117/12.896483.

101. J. Zhou et al., "Fabrication of low-loss, single-mode-channel waveguide with DNACTMA biopolymer by multistep processing technology," Opt. Lett. 35, 1512-1514 (2010), http://dx.doi.org/10.1364/OL.35.001512.

102. P. Gupta et al., "DNA-ormocer-based biocomposite for fabrication of photonic structures," Appl. Phys. Lett. 88, 213109 (2006), http://dx.doi.org/10.1063/1.2204648.

103. A. V. Pinheiro et al., "Challenges and opportunities for structural DNA nanotechnology," Nat. Nanotechnol. 6, 763-772 (2011), http://dx.doi.org/10.1038/nnano.2011.187.

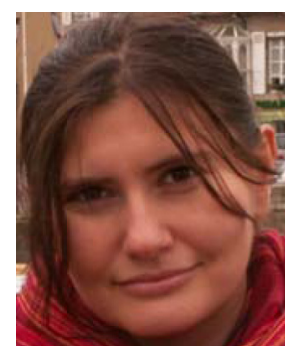

Katarzyna Matczyszyn received her PhD in physical chemistry in 2003 from Wroclaw University of Technology (WUT), Poland. After her thesis defence she spent a few months in the group Cliq of J.M. Otón Sánchez at Universidad Politécnica de Madrid working with liquid crystals for displays, then as a post-doc at Commissariat Energie Atomique CEA Saclay with C. Fiorini and F. Kajzar studying nonlinear optical properties of azobenzenes. She worked also at Université Pierre et Marie Curie (UPMC) in Paris with A-J. Attias and J-L. Fave (Institute des NanoScience de Paris) on time-of-flight measurements in liquid crystals. As an ATER at Ecole Normale Supérieure in Cachan she collaborated with K. Nakatani and J. Zyss on two-photon imaging of DNA and photochromic nanocrystals. In 2009 she was an invited researcher at Australian National University in Canberra where she gained her knowledge about Z-scan techniques with M. Samoc. Her scientific interests cover DNA in the liquid crystal phase, photochromic materials (also as DNA markers), liquid crystals for nonlinear optics and electronics, and functionalisation of fluorescent nanoparticles for biodetection. She is strongly involved in the Erasmus Mundus Master of Excellence Programme "Monabiphot" between France (ENS de Cachan), Spain (Universidad Complutense), and Poland (UW, WUT). She is a co-founder and scientific tutor of the international students association "PhoBia - Photonics Bionanotechnology Association." Currently she is working in the group lead by Professor Samoc at WUT and collaborating strongly with ENS de Cachan and UPMC in Paris. She is an author of about 30 scientific papers.

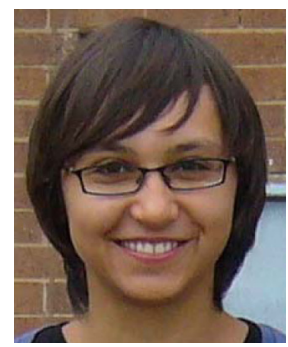

Joanna Olesiak-Banska graduated from the Erasmus Mundus Master Programme in molecular nano- and biophotonics for telecommunications and biotechnologies "Monabiphot" and received the title of MSc Eng. of biotechnology from Wroclaw University of Technology, Poland and MSc of physics from Ecole Normale Supérieure (ENS) de Cachan, France. She is an author of 8 publications. Now she is finishing her $\mathrm{PhD}$ in the group lead by professor Marek Samoc at Wroclaw University of Technology, in collaboration with Laboratoire de Photonique Quantique et Moléculaire, at ENS de Cachan. Her doctoral research is concerned with the application of two-photon microscopy to imaging of DNA self-assembly and investigation of nonlinear optical properties of dye molecules and nanoparticles applied as DNA markers. 